\title{
ANNOUNCEMENTS/
}

\section{APRIL MEETINGS}

April 16-18. UKFCC Laboratory Meeting on Research in National Culture Collections (and training course on Maintenance of Microorganisms). Leicester, U.K. Info: J. V. Lee, PHLS CAMR, Porton Down, Salisbury SP4 0JG, U.K.

April 17-19. International Symposium on Analytical Methods and Problems in Biotechnology. Noordwijkerhout, The Netherlands. Info: W. A. Scheffers, Symposium on Analytical Methods and Problems in Biotechnology, Delft University of Technology, Laboratory of Microbiology, Julianalaan 67A, NL-2628 BC Delft, The Netherlands

\section{MAY METINGS}

May 2-4. Molecular Basis of Cellular Recognition. Bethesda, MD. Info: J. Michael Poston, National Institutes of Health, Building 3, Room 216, Bethesda, MD 20205

May 7-8. Advances in Carriers and Adjuvants for Veterinary Biologics. Ames, IA. Info: R. M. Nervig, National Veterinary Services Laboratories, P.O. Box 844, Ames, IA 50010

May 8. Biochemistry of Mixed Cultures. London, U.K. Info: Society of Chemical Industry, 14/15 Belgrave Square, London SWIX 8PS, U.K.

May 8-11. International Seminar on Anaerobic Digestion and Carbohydrate Hydrolysis of Waste. Luxemburg. Info: P. P. Rotondo, Commission of the European Communities, DG XIII-A2, P.O. Box 1907, L-2920 Luxembourg

May 14-18. Research Conference on Plasmids in Bacteria. Urbana-Champaign, IL. Info: Carol Madison, 116 Illini Hall, $725 \mathrm{~S}$. Wright St., University of Illinois, Champaign, Il 61820

May 15-17. Biotech Europe. London, U.K. Info: Biotech Europe, Online Conferences Ltd., Pinner Green House, Ash Hill Drive, Pinner HA5 2AE, Middlesex, U.K.

May 15-18. Sixth Symposium on Biotechnology for Fuels and Chemicals. Gatlinburg, TN. Info: Charles D. Scott, Sixth Symposium on Biotechnology, Oak Ridge National Laboratory, P.O. Box X, Oak Ridge, TN 37830
May 21-25. International Conference on Microbial Enhancement of Oil Recovery. Conroe, TX. Info: Erle Donaldson, U.S. Department of Energy, P.O. Box 1398, Bartlesville, OK 74005

May 22-23. Stony Brook Symposium: Molecular Basis of the Diagnosis and Treatment of Human Disease. Stony Brook, NY. Info: Stony Brook Symposium, Dept. of Biochemistry, SUNY, Stony Brook, NY 11794

May 30-June 3. Sixth Conference on Macromolecular Synthesis: Gene Expression. Hamburg-Blankenese, F.R.G. Info: D. Richter, Physiologisch-Chemisches Institut der Universität Hamburg, Martinistraße 52, 2000 Hamburg 20, F.R.G.

\section{JUNE METINGS}

June 3-7. Tissue Culture Association (35th Annual Meeting). Houston, TX. Info: James R. Florini, Biology Dept., Syracuse University, 108 College Place, Syracuse, NY 13210

June 4-8. Second International Symposium on Molecular Genetics of the Bacteria-Plant Interaction. Ithaca, NY. Info: A. A. Szalay, Boyce Thompson Institute, Cornell University, Tower Road, Ithaca, NY 14853

June 7-10. Fourth International Symposium on Rapid Methods and Automation in Microbiology and Immunology. Berlin, F.R.G. Info: K.-O. Habermehl, Institute of Clinical and Experimental Virology of the Free University of Berlin, Hindenburgdamm 27, D-1000 Berlin 45, F.R.G.

June 10-14. 10th International Congress on Animal Reproduction and Artificial Insemination. UrbanaChampaign, IL. Info: Carol D. Holden, Conferences and Institutes, 116 Illini Hall, University of Illinois at Urbana-Champaign, 725 South Wright St., Champaign, IL 61820

June 11-15. 28th Wind River Conference on Genetic Exchange. Saugerties, NY. Info: Rivka Rudner, Dept. of Biological Sciences, Hunter College of CUNY, 695 Park Avenue, Box 1377, New York, NY 10021

June 18-20. 101st Society for General Microbiology Ordinary Meeting. Sheffield, U.K. Info: R. C. W. Berke- ley, Dept. of Microbiology, The Medical School, University Walk, Bristol BS8 ITD, U.K.

June 18-21. Bio-Energy 84. Gothenburg, Sweden. Info: The Bio-Energy Council, 1625 I St., N.W., Suite 825A, Washington, D.C. 20036-3190

June 25-29. Chemrawn III: Resource Material Conversion to Meet Future Needs. The Hague, The Netherlands. Info: Congress Bureau, QTL Convention Services, Keizersgracht 792, 1017 EC, Amsterdam, The Netherlands

\section{JULY METTINGS}

July 9-13. Sixth International Symposium on Yeast. Montpellier, France. Info: Midi Contacts, Le Capoulie, Rue Maguelone, BP 1041, 34006 Montpellier, Cedex, France

July 16-20. Cellucon '84-International Conference on the Chemistry, Biochemistry and Applications of Cellulose and its Derivatives. Wrexham, U.K. Info: Conference Secretariat, Cellucon '84, Research Division, The North E. Wales Institute, Kelsterton College, Deeside, Clwyd CH5 4BR, U.K.

July 16-20. UNESCO Sponsored Symposium on Biomass Conversion Technology: Principles \& Practice. Waterloo, Ont., Canada. Info: M. Moo-Young, Institute for Biotechnology Research (E-1), University of Waterloo, Waterloo, Ont., Canada, N2L $3 G 1$.

July 16-20. Society for Applied Bacteriology Meeting: Microbiological Aspects of Water Management. Lancaster, U.K. Info: R. W. A. Park, Microbiology Dept., University of Reading, London Road, Reading RG1 $5 \mathrm{AQ}$

July 18-20. Biochemical Society Annual General Meeting. Leeds, U.K. Info: Doris E. Herriott, Meetings Officer, The Biochemical Society, 7 Warwick Court, High Holborn, London WC1R 5DP, U.K.

July 29-August 4. Eighth International Biophysics Congress. Bristol, U.K. Info: Congress Secretariat, Eighth International Biophysics Congress, Meon Conference Services, Petersfield, Hampshire GU32 3JN, U.K. 